\title{
Large-Area, Low-Noise, High Speed, Photodiode-Based Fluorescence Detectors with Fast Overdrive Recovery
}

\author{
S. Bickman, D. DeMille \\ Yale University, Physics Department, PO Box 208120, SPL 23, New Haven, CT, 06520
}

\begin{abstract}
Two large-area, low noise, high speed fluorescence detectors have been built. One detector consists of a photodiode with an area of $28 \mathrm{~mm} \times 28 \mathrm{~mm}$ and a low noise transimpedance amplifier. This detector has a input light-equivalent spectral noise density of less than $3 \mathrm{pW} / \sqrt{\mathrm{Hz}}$, can recover from a large scattered light pulse within $10 \mu \mathrm{s}$, and has a bandwidth of at least $900 \mathrm{kHz}$. The second detector consists of a $16 \mathrm{~mm}$ diameter avalanche photodiode and a low-noise transimpedance amplifier. This detector has an input light-equivalent spectral noise density of $0.08 \mathrm{pW} / \sqrt{\mathrm{Hz}}$, also can recover from a large scattered light pulse within $10 \mu \mathrm{s}$, and has a bandwidth of $1 \mathrm{MHz}$.
\end{abstract}

\section{Introduction}

Two large-area, high speed, photodiode-based fluorescence detectors have been built to detect fluorescence from molecules excited via a pulsed laser system. Both detectors have a large area to allow for collection of light from a large solid angle and are fast to allow for good time resolution of the fluorescence. Since the high intensity of pulsed laser systems inevitably scatters a significant number of photons into the detector, both detectors are designed to quickly recover from a large pulse of light that would otherwise saturate the detector for the duration of the fluorescence signal.

While photomultiplier tubes (PMTs) can also be used to measure fluorescence after excitation with a pulsed laser, photodiodes and avalanche photodiodes (APDs) have several advantages. Photodiodes typically have a much higher quantum efficiency (q.e.) than PMTs. For example, at $550 \mathrm{~nm}$ (where this detector is used) PMTs have q.e. $\approx 20 \%$ while Silicon PIN photodiodes have q.e. $\approx 85 \%$ and the APD used in this detector has q.e. $\approx 80 \%$. Furthermore, photodiodes have a more linear response to light intensity than PMTs and can withstand higher sustained fluxes.

One of these detectors will be used in an experiment that intends to improve the sensitivity to an electron electric dipole moment (EDM)[1]. The experiment detects small energy shifts in an excited state of $\mathrm{PbO}$ that would result from a non-zero EDM. The energy shifts are measured with quantum beat spectroscopy [2], which in this case appears as a sinusoidal modulation at $200-500 \mathrm{kHz}$ superimposed on an exponential decay due to the spontaneous emission of an excited state of $\mathrm{PbO}[3]$. In order to maximize the solid angle of detection, large area photodiodes or APDs are used. The Hamamatsu S3584-08 silicon PIN photodiode has an area of $28 \mathrm{~mm} \times 28 \mathrm{~mm}$ and the Advanced Photonix 630-70-73-500 APD has a diameter of $16 \mathrm{~mm}$.

While PMTs have an intrinsic nearly noise-free gain stage, PIN photodiodes do not and the gain in
APDs is smaller than the desired gain. Thus, low noise transimpedance preamplifiers are necessary for the photodiode-based detectors. There were three requirements for the design of the preamplifiers in our experiment. First, the intrinsic noise of the amplifiers must be less than the anticipated shot noise, so that the amplifier noise will not significantly contribute to the overall noise on the detected signal. Second, since the photodiode or APD is exposed to a large pulse of scattered light from the excitation laser, the preamplifier must be able to recover quickly from such a pulse. We specifically require recovery in $\lesssim 10 \mu$ s, since the excited state has a lifetime of $50 \mu \mathrm{s}$ under the current conditions. Finally, to allow for unattenuated detection of quantum beats at $\sim 500 \mathrm{kHz}$, the preamplifier must have a bandwidth of $\gtrsim 1 \mathrm{MHz}$.

\section{Low noise amplification}

The noise of a transimpedance amplifier can be modelled as shown in figure 114]. All noise components will be calculated at the output of the transimpedance amplifier. A real photodiode can be modelled as an ideal photodiode in parallel with a capacitor $C_{j}$ and a shunt resistor $R_{j}$, plus resistance $R_{s}$ in series with the other components. $C_{f}$ and $R_{f}$ are the feedback capacitance and resistance and $C_{a}$ is the amplifier input capacitance.

All of the resistances in this model have intrinsic Johnson noise. The voltage noise spectral density $e_{J}$ across a resistance $R$ is given by $e_{J}=\sqrt{4 k_{B} T R}$. At the output of the amplifier, the Johnson noise of $R_{j}$ contributes

$$
e_{o u t, J j}=\frac{\frac{R_{f}}{1+s C_{f} R_{f}}}{R_{j}+R_{s}} e_{J j},
$$

where $C_{i}=C_{f}+C_{a}$ and $s=i \omega$. At the output of the amplifier, the Johnson noise from $R_{s}$ gives

$$
e_{o u t, J s}=\left(1+\frac{\frac{R_{f}}{1+s C_{f} R_{f}}}{R_{s}+\frac{R_{j}}{1+s C_{i} R_{j}}}\right) e_{J s}
$$




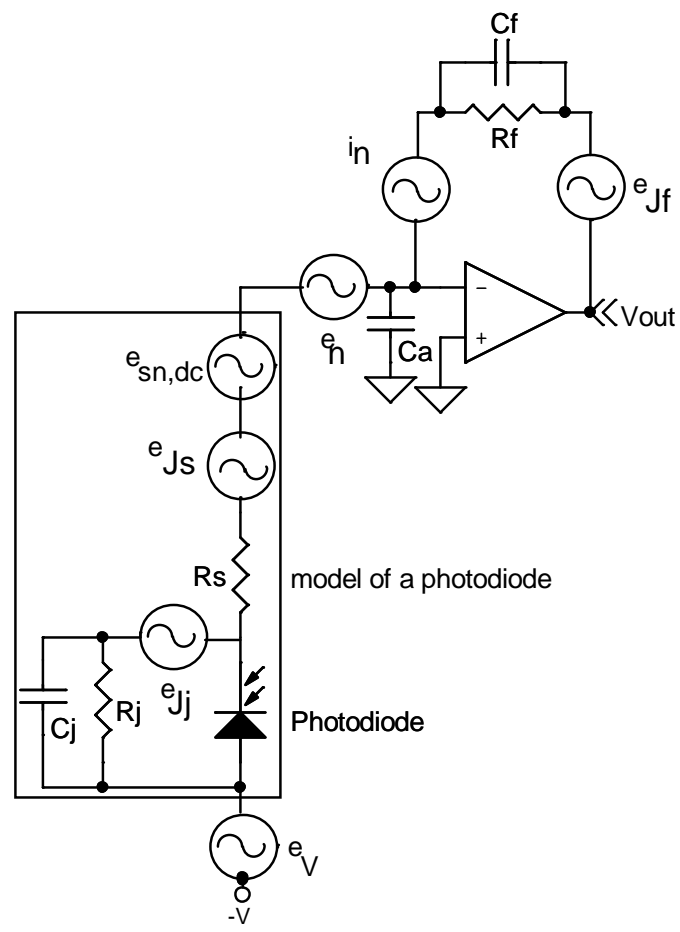

Figure 1: Noise model of a transimpedance amplifier. Here, a real photodiode is modelled as an ideal photodiode in parallel with a junction capacitance $C_{j}$, a shunt resistance $R_{j}$ and a series resistor $R_{s}$.

and the Johnson noise from $R_{f}$ gives

$$
e_{\text {out }, J f}=\frac{e_{J f}}{1+C_{f} R_{f} s} .
$$

In addition to Johnson noise, we must consider the voltage and current noise at the input of the amplifier. At the output of the amplifier, the input voltage noise of the amplifier $e_{n}$ contributes

$$
e_{\text {out }, n}=\left(1+\frac{\frac{R_{f}}{1+s C_{f} R_{f}}}{R_{s}+\frac{R_{j}}{1+s C_{i} R_{j}}}\right) e_{n} .
$$

The input current noise $i_{n}$, at the output of the amplifier, gives

$$
e_{o u t, i}=\frac{R_{f} i_{n}}{1+C_{f} R_{f} s} .
$$

In this application, it is necessary to keep the total electronic noise less than the shot noise. The expected signal size is $\dot{N}=2 \times 10^{8}$ photoelectrons, in an exponential decay with a time constant $\tau$ of $50 \mu \mathrm{s}$. For a signal current $I_{s i g}=\frac{\dot{N} e}{\tau} \exp (-t / \tau)$, the current noise spectral density at the input of the detector is

$$
i_{s n}=\sqrt{2 e I_{s i g}} \sqrt{F} G .
$$

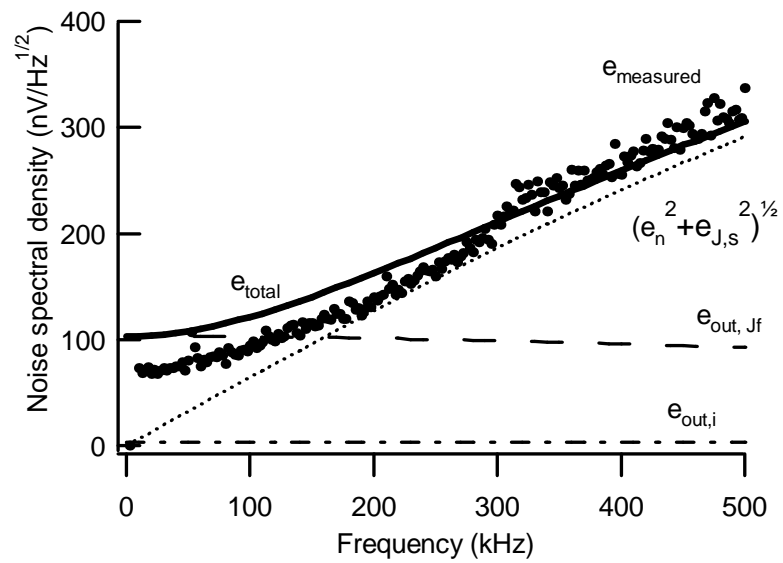

Figure 2: Graph of measured and predicted noise referred to the output of the preamplifier for the PIN photodiode. For comparison, the predicted shot noise in our signals is $420 \mathrm{nV} / \sqrt{H z}$.

Here $G$ is the intrinsic gain of the detector; $G=1$ for a PIN photodiode, while $G=200$ for the APD used here. Also, $F$ is an additional noise factor associated with the gain process. For the PIN photodiode, $F=1$, while for an APD $F \geq 2[5]$. In the case of the APD used here, this additional noise was measured as indistinguishable from $\mathrm{F}=2$. At the output of the amplifier, this becomes

$$
\delta V_{s n}=R_{f} \frac{\sqrt{2 e I_{s i g}}}{1+C_{f} R_{f} s} \sqrt{F} G .
$$

\section{A PIN Photodiode}

In the photodiode transimpedance amplifier, the feedback resistor was chosen to be $R_{f}=600 \mathrm{k} \Omega$. In this case, the shot noise on the signal (at low frequencies) is $\delta V_{\text {sig,sn }}=0.3 \mu \mathrm{V} \exp (-t / 2 \tau)$ (also at low frequencies) which is larger than the Johnson noise of the feedback resistor for the first $100 \mu \mathrm{s}$ of the decay since $e_{\text {out }, J f}=0.1$ $\mu \mathrm{V}$ when $\omega \ll \frac{1}{R_{f} C_{f}}$. Having chosen $R_{f}=600 \mathrm{k} \Omega$, it is possible to make some estimates of which noise terms are important. The Hamamatsu S3584-08 photodiode used to detect the signals has a capacitance $C_{j} \approx 200 \mathrm{pF}$ when reverse biased and resistances $R_{j} \sim 5 \mathrm{G} \Omega$ and $R_{s} \sim 5 \Omega$. $C_{f}$ is approximately $0.3 \mathrm{pF}$ in order to maintain the desired bandwidth. With these values, the significant noise sources are $e_{J s}, e_{J f}$, and $e_{n}$, which all contribute on the order of $10^{-7} \mathrm{~V} / \sqrt{\mathrm{Hz}}$ at the output. At high frequencies, $e_{\text {out }, n} \approx e_{n} \frac{C_{i}}{C_{f}}$, so it is necessary to keep $e_{n} \ll 1$ $\mathrm{nV} / \sqrt{H z} . e_{J j}$ and $i_{n}$ contribute very little to the noise (see figure 2). In order to control the input voltage and current noise of the amplifier at the desired level, it is necessary to use a low noise JFET as the the input stage of the amplifier. Most low-noise operational amplifiers have 


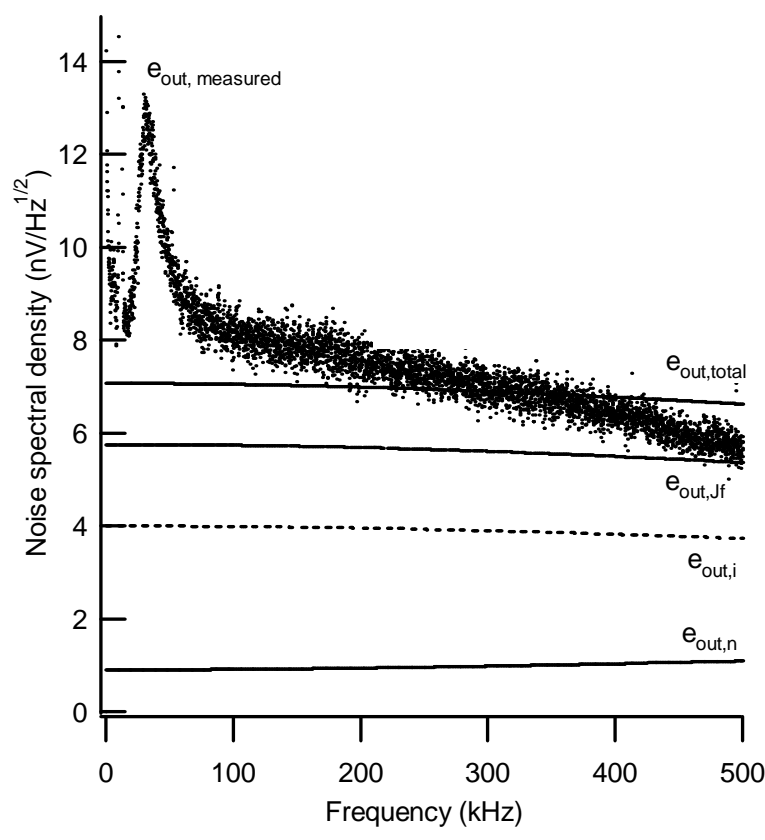

Figure 3: Graph of measured and predicted noise referred to the output of the preamplifier for the APD. For comparison, the predicted shot noise on our signal is $70 \mathrm{nV} / \sqrt{\mathrm{Hz}}$. The low frequency noise spikes appear to be a result of poor shielding on the APD.

an input voltage spectral noise which is too high for this application. Each of the IF9030 JFETs used here has $e_{n}=$ $0.5 \mathrm{nV} / \sqrt{\mathrm{Hz}}$. These JFETs do not have enough gain to achieve the desired transimpedance gain of $\frac{V_{\text {out }}}{I_{\text {in }}}=6 \times 10^{5}$ $\Omega$, so this stage is followed by an operational amplifier[6] The JFET input stage provides a low-noise amplification stage before the noisier second stage op-amp. The gain of the JFET amplifier is sufficiently large that the input noise of the op-amp is negligible.

The input voltage noise $e_{n}$ can be further reduced by putting $m$ JFETs in parallel resulting in a combined noise of $e_{n}=e_{J F E T} / \sqrt{m}$. In principle, many JFETs can be used to improve the noise of the amplifier, but in practice there are two limitations. The ultra-low noise JFETs used here have a significant gate-source capacitance $C_{J F E T}$. Since the noise at the output of the amplifier at high frequencies scales as $e_{n} C_{a} \propto \frac{1}{\sqrt{m}}\left(C_{j}+m C_{J F E T}\right)$, the number of parallel JFETs has an optimum value $m \approx C_{j} / C_{J F E T}$ [7]. Also, since $e_{J, s}$ is of the same order as $e_{n}$, there is little improvement to the noise once $e_{n} \lesssim e_{J, s}$.

Requirements for the second stage of amplification were also stringent. At high frequencies the closed-loop voltage gain of the entire preamplifier is $G_{v, c l} \approx \frac{C_{i}}{C_{f}} \sim$ 1000. However, the voltage gain of the JFET front-end is only $G_{1} \sim 60$, determined by the JFET transconductance of $g_{m}=0.02 \mathrm{~S}$ and the drain resistance of $3 \mathrm{k} \Omega$. The
Miller effect significantly reduces the gain of the JFET stage at high frequencies, so a cascode configuration was used to reduce this effect[8]. Even with the cascode, the second stage amplifier must have a high gain and wide bandwidth so that the open loop gain is large enough. We use the Analog Devices AD797, which which has a gain bandwidth product GBW of $110 \mathrm{MHz}$, and has $e_{n 2}=0.9$ $\mathrm{nV} / \sqrt{\mathrm{Hz}}$. In addition to the high GBW of the AD797, this op-amp was chosen for its fast recovery from overdrive, which allows it to quickly recovery from the scattered light pulse.

\section{B APD}

The noise design of the transimpedance amplifier for the APD is much less stringent since the bias voltage is chosen so the APD gain $G \approx 200$. The shot noise at the output of the APD is thus $\sqrt{2} G$ larger than the noise in the PIN photodiode. We choose $R_{f}=2 \mathrm{k} \Omega$ in the transimpedance amplifier for the APD, resulting in shot noise at the output of the amplifier $\delta V_{s n}=100 \mathrm{nV} / \sqrt{\mathrm{Hz}}$. Having chosen $R_{f}$ and knowing that $C_{j}=140 \mathrm{pF}$ for this APD, it is possible to make some estimates of which noise terms are significant. We find that $R_{j}$ and $R_{s}$ have insignificant noise contributions. Since the noise requirements are much less strict for this amplifier, no front-end JFET was used. Instead the transimpedance amplifier is made from a single opamp, the AD797, which has again been chosen for its high GBW and fast overdrive recovery time. The AD797 has $e_{n}=0.9 \mathrm{nV} / \sqrt{\mathrm{Hz}}$ and $i_{n}=2.0 \mathrm{pA} / \sqrt{\mathrm{Hz}}$. At the output of the transimpedance amplifier, $e_{\text {out }, J f} \sim 5.8 \mathrm{nV} / \sqrt{\mathrm{Hz}}$. The voltage noise is almost insignificant since $e_{\text {out }, n} \sim$ $1.3 \mathrm{nV} / \sqrt{\mathrm{Hz}}$. The current noise is the largest expected noise contribution at $e_{\text {out }, i} \sim 3.7 \mathrm{nV} / \sqrt{\mathrm{Hz}}$. The various noise contributions along with the measured noise are shown in figure 3

There are two other noise sources that are significant with the APD detector, but not the photodiode detector. The first is the shot noise in the dark current of the APD $e_{s n, d c}$. In this case, this shot noise is negligible compared to the shot noise on the expected signal. The second is the noise on the bias voltage for the APD. The photodiode bias voltage was supplied by batteries, but the necessary high bias voltage for the APD makes it difficult to supply with batteries. A high voltage power supply was used and filtered to provide acceptable noise characteristics for the APD detector.

\section{Recovery time from scattered light pulse}

Many experiments observe the fluorescence from atoms or molecules that are excited with a pulsed laser. These high 


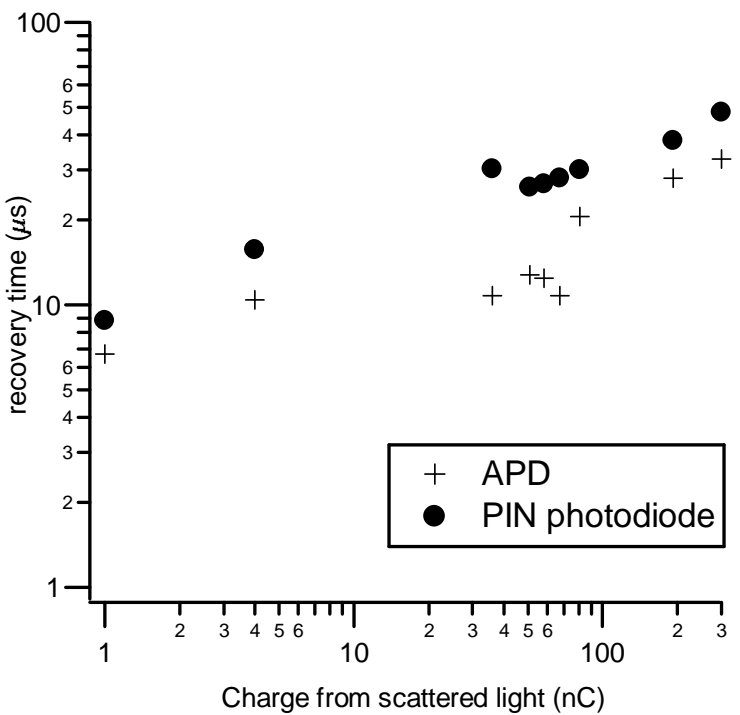

Figure 4: Graph of the recovery time of the detectors vs the size of the scattered light pulse.

intensity beams scatter photons into the detector causing a large, but temporally short burst of photoelectrons. In many cases, this scattered light pulse is several orders of magnitude larger than the expected signal size, and can easily saturate the detector system. In our case, the transimpedance amplifier must be able to recover from this scattered light pulse, which is injected in a $\sim 5$ ns duration, quickly in order to observe the spontaneous fluorescence. We define the recovery time as the time that it takes the amplifier to ring down to $1 / \mathrm{e}$ of the saturated value.

The recovery time of the amplifier depends on the size of the scattered light pulse. For this experiment, it was necessary to choose optical filters that reduced the signal size, but also reduced the scattered light. The two filtering options we considered allowed for a scattered light pulse that injected a charge of either $220 \mathrm{nC}$ or $1.5 \mathrm{nC}$ at the expense of a factor of 3 smaller desirable signal in the latter case. The photodiode and APD both took more than $50 \mu \mathrm{s}$ to recover from the $220 \mathrm{nC}$ pulse and $\approx 10 \mu$ s to recover from the $1.5 \mathrm{nC}$ pulse. The recovery time as a function of injected charge from scattered light is shown in figure 4 for both detectors.

Three changes were made to the design of the transimpedance amplifiers to improve the recovery time. First, clamping diodes were placed between the output of the photodiode or APD and ground to shunt large input light signals to ground. Small signal and Schottky diodes were used and both kinds were chosen for their low capacitance so as not to significantly contribute to $C_{i}$. The Schottky diodes were chosen for their low forward voltage drop, and the small signal diodes were chosen for their higher current ratings. These diodes are placed in opposite di-

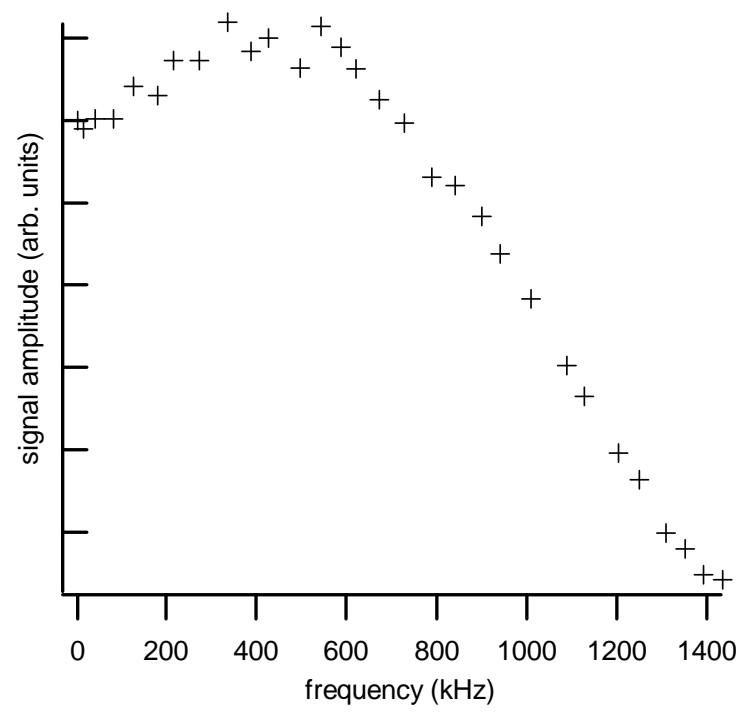

Figure 5: Measured frequency response of the PIN photodiode transimpedance amplifier.

rections to damp additional electronic ringing due to the scattered light pulse.

The second and third changes were only applied to the photodiode amplifier. Here, diodes were placed in the feedback loop to allow for a low resistance path when the amplifier was saturated. These diodes were not necessary in the APD amplifier because $R_{f}$ is much smaller. The cascode in the JFET stage improved the recovery time by increasing the bandwidth of this stage of the amplifier.

Preliminary circuit designs used two additional methods of improving the recovery time, but these methods were not successful enough to be used in the final design. The first method was to add a power booster in the feedback loop for the photodiode detector. This power booster was able to source more current through the diodes in the feedback loop of this detector in an attempt to shorten the recovery time. In this application, it was difficult to implement the power booster as it made the feedback traces larger, and $C_{f}$ difficult to minimize. The second method was to add an additional photodiode at the input of the transimpedance amplifier with opposite orientation to the detecting photodiode. A laser diode shone into the additional diode providing some charge cancellation during the scattered light pulse. However, the improvement in recovery time was found to be negligible.

\section{Frequency Response}

In order to achieve the desired bandwidth of $\sim 1 \mathrm{MHz}$ with a feedback resistor of $600 \mathrm{k} \Omega$ in the PIN photodiode transimpedance amplifier, it is necessary to keep the feedback capacitance less than $0.26 \mathrm{pF}$. Since the diodes used to de- 


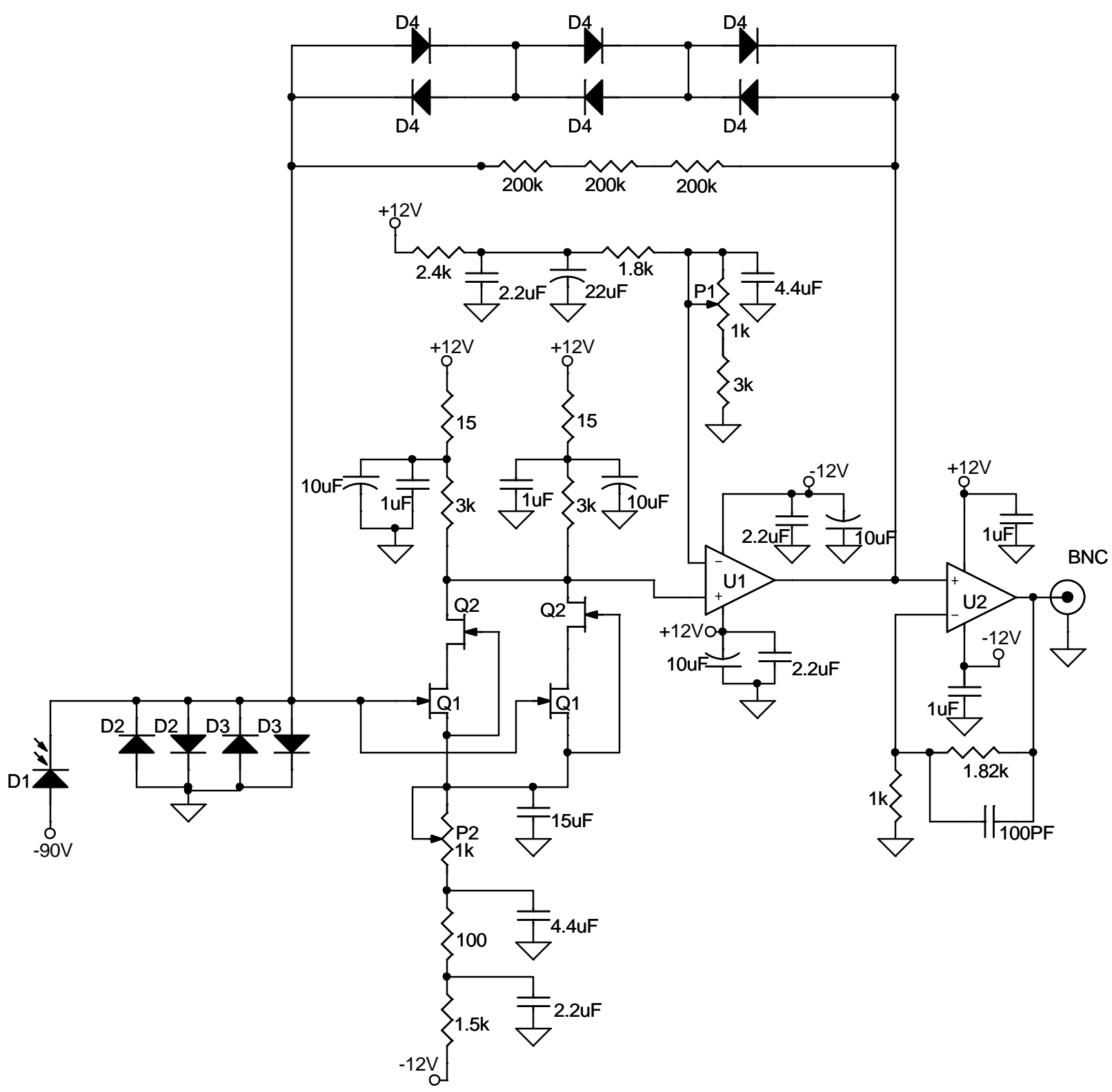

Figure 6: Circuit diagram for the PIN photodiode detector. D1 is the large-area Hamamatsu S3584-08 photodiode. D2 are BAS70-04 Schottky diodes chosen for their small capacitance and low forward voltage. D3 are small signal HSMP-3822-BLK diodes, which allow for more current conductance than the Schottky diodes. D4 are also HSMP3822-BLK diodes, chosen for their low capacitance. When the preamplifier is saturated, these diodes provide a low resistance path in the feedback loop. Since the JFETs Q1 and Q2 drift with temperature, P1 and P2 are used to finely adjust the voltages at the inputs of U1 so that the two inputs are at the same DC voltage. Q1 is chosen for its low voltage noise, and is an IF9030 from InterFET. Q2 is used in a cascode configuration to increase the speed of the JFETs and is a 2N4856A from InterFET. U1 is an AD797, which was chosen for its high speed, low noise, and fast overdrive recovery. $\mathrm{U} 2$ is an AD829, which is a cable driver chosen for its fast overdrive recovery. 


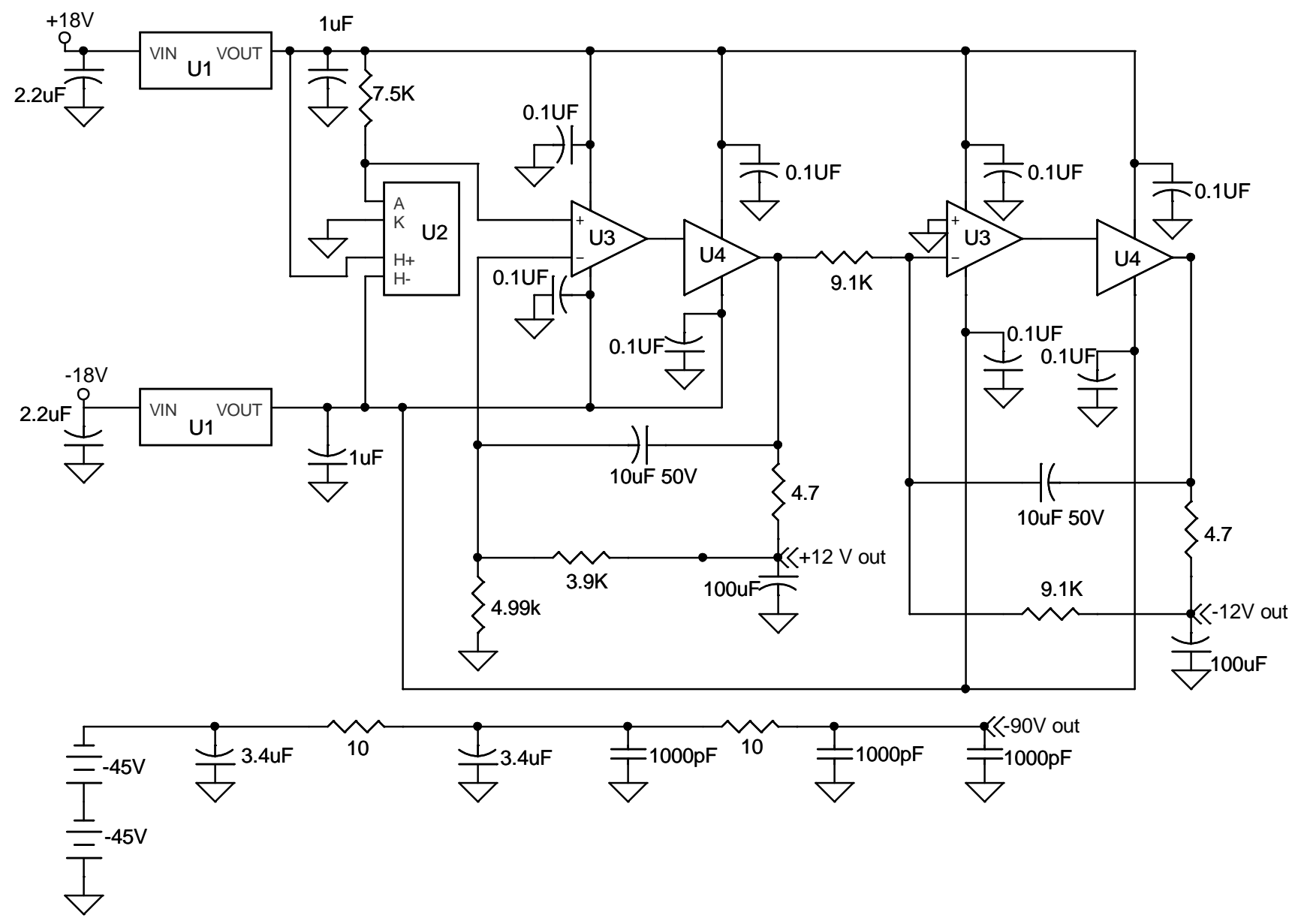

Figure 7: Power supplies for preamplifier. The $\pm 18 \mathrm{~V}$ power supplies powering these components are powered by a PowerOne AC/DC converter. This circuit can supply $200 \mathrm{~mA}$ continuously, and up to $500 \mathrm{~mA}$ for short times. The noise on these power supplies is less than $2 \mathrm{nV} / \sqrt{\mathrm{Hz}}$ for all frequencies between $10 \mathrm{~Hz}$ and $1 \mathrm{MHz}$. U1 are 7815 and 7915 voltage regulators. U2 is a LM399H voltage reference, which was chosen for its stability. U3 are low-noise OP77 op-amps, and U4 is a BUF634 power-boosting amplifier. The two $-45 \mathrm{~V}$ power supplies are batteries. 
crease the scattered light recovery time have a combined capacitance of $0.2 \mathrm{pF}$, it is necessary to keep all other possible capacitances extremely low. For this reason, three $200 \mathrm{k} \Omega$ resistors were used in series to create the feedback resistor. Additionally, the circuit board was designed to keep the traces relating to the components in the feedback loop as short as possible by placing feedback components on the opposite side of the circuit board from the amplifying components. Ground planes were placed on all available surfaces on the circuit board and two inner layers of the circuit board were also grounded. The $3 \mathrm{~dB}$ bandwidth of the circuit was measured as $\approx 900 \mathrm{kHz}$, implying $C_{f} \approx$ $0.3 \mathrm{pF}$ (see figure 5).

The smaller $R_{f}$ in the APD amplifier makes it much less sensitive to frequency limitations and this amplifier could be made to have a bandwidth of at least $10 \mathrm{MHz}$ if necessary. Our present APD detector has a bandwidth of $1 \mathrm{MHz}$.

\section{Final Design}

The final design incorporated all of the elements discussed above. The circuit diagram for the PIN photodiode detector is shown in figure 6 The circuit boards were designed with great care to minimize the path lengths at the input of the amplifier. These short paths reduce capacitive effects and noise pickup from other sources. Surface mount components were used wherever possible to reduce lead size. All cables leading to or from the circuit board are made of coaxial cable. The power supplies for the photodiode detector were also constructed to minimize noise. The design of the power supplies is shown in figure 7

The final design for the APD detector is shown in figure 8 The power supply for the bias voltage was filtered to remove high frequency noise components.

\section{Summary}

The large-area $\left(7.8 \mathrm{~cm}^{2}\right)$ PIN photodiode detector described here has an input light-equivalent noise of less than $3 \mathrm{pW} / \sqrt{\mathrm{Hz}}$ at a wavelength of $550 \mathrm{~nm}$, can recover in 10 $\mu$ s from large scattered light pulses that rapidly inject 1.5 $\mathrm{nC}$ of charge, and has a bandwidth of more than $900 \mathrm{kHz}$. The noise was minimized by using a large feedback resistance to minimize Johnson noise, JFETs as the first stage of the amplifier to $e_{n}$, and low noise power supplies. The recovery time from the scattered light was reduced by using clamping diodes on the input and in the feedback, and with a cascode configuration on the JFET front end. The bandwidth was achieved by minimizing all capacitances.

The final design for the large-area $\left(2.0 \mathrm{~cm}^{2}\right)$ APD detector has an input light-equivalent noise of $0.08 \mathrm{pW} / \sqrt{\mathrm{Hz}}$, can recovery quickly from large scattered light pulses and

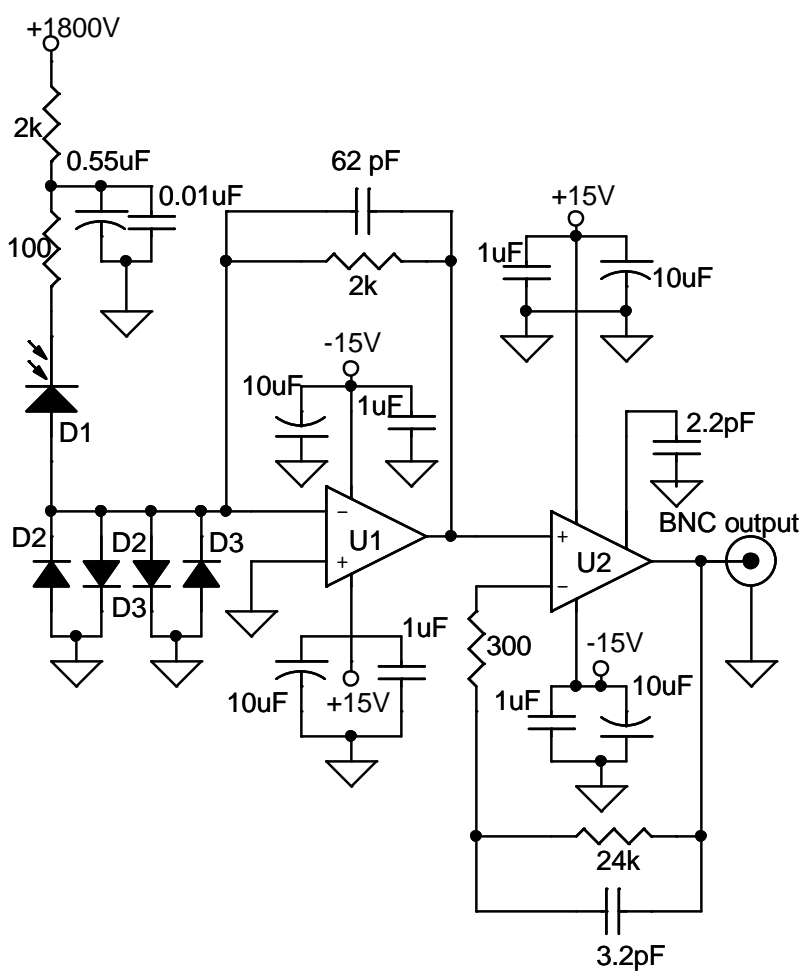

Figure 8: Circuit Diagram for the APD detector. D1 is the APD, and is the 630-70-73-500 from Advanced Photonix, chosen for its large area. D2 is a BAS70-04 Schottky diode, chosen for its low forward voltage and low capacitance. D3 is a HSMP-3822-BLK diode chosen for low capacitance. U1 is an AD797, chosen for low-noise, high speed and fast overdrive recovery. U2 is an AD829, a high speed cable driver with fast overdrive recovery. 


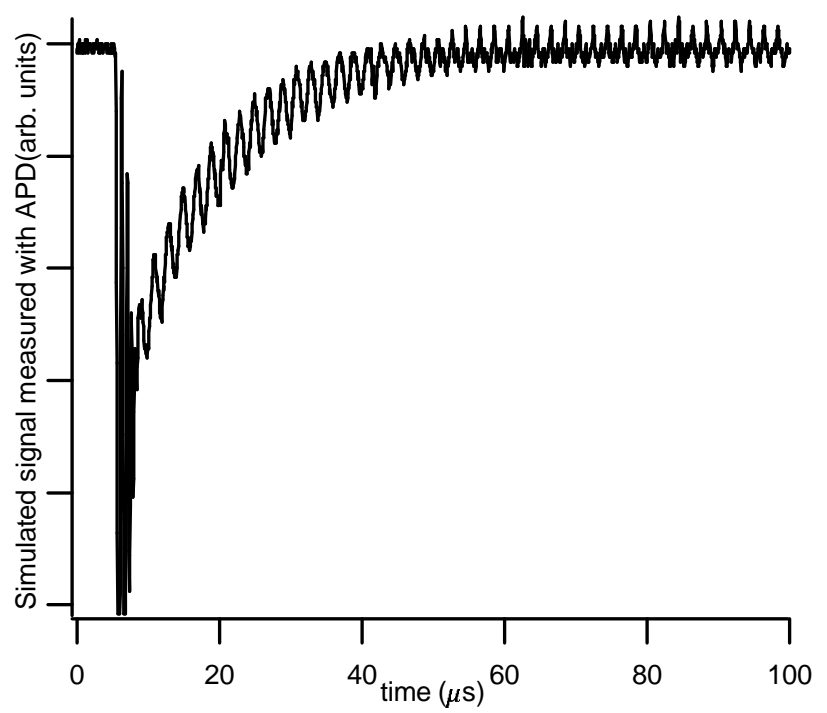

Figure 9: Simulated quantum beats at $500 \mathrm{kHz}$ measured with the APD detector and scattered light equivalent to 1.5 $\mathrm{nC}$ of injected charge.

easily has a bandwidth of $1 \mathrm{MHz}$. Quantum beats were observed with this APD detector; however, the signals in the current experimental configuration are much smaller than ultimately anticipated. In figure 9 simulated signals at the anticipated level of $0.6 \mu \mathrm{A}$, with quantum beats at 500 $\mathrm{kHz}$, are shown as measured with the APD. These signals were simulated with an LED driven by an arbitrary waveform generator programmed to simulate the beat signal. The trace in figure 9 includes scattered light equivalent to $1.5 \mathrm{nC}$ of charge injection.

\section{Acknowledgements}

We are grateful for the support of NSF Grant No. PHY0244927, and the David and Lucile Packard Foundation. We thank David Kawall and Valmiki Prasad for helpful discussions.

\section{References}

[1] D. Kawall, F. Bay, S. Bickman, Y. Jiang, and D. DeMille Phys. Rev. Lett. 92, 133007 (2004)

[2] S. Haroche in High-Resolution Laser Spectroscopy edited by K. Shimoda (Springer-Verlag, Berlin, 1976), Chap. 7, pp 253-313.

[3] D. DeMille, F. Bay, S. Bickman, D. Kawall, D. Krause, Jr., S. E. Maxwell, and L. R. Hunter, Phys. Rev. A 61, 052507 (2000).
[4] J. Graeme, Photodiode Amplifiers: Op-Amp Solutions. McGraw-Hill, New York 1996.

[5] Ammon Yariv. Optical Electronics, Third Edition, CBS College Publishing, USA (1985).

[6] D. Yvon, A. Cummings, W. Stockwell, P. Barnes, C. Stanton, B. Sadoulet, T. Schutt, C. Stubbs, Nucl. Instr. and Meth. in Phys. Res. A 368 (1996) 778-788.

[7] Paul Horowitz, and Winfield Hill. The Art of Electronics, Second Edition, Cambridge University Press, USA (1998).

[8] Steven R. Jefferts, and F.L. Walls. Rev. Sci. Instru. 60 (1989) 1194-1196. 